\title{
Vergünstigungen im Arzneimittelhandel: Noch mehr Empfehlungen des BSV
}

\author{
FMH-Zentralvorstand
}

Nach einem ersten unbrauchbaren Anlauf im Dezember 2001 (Schweiz Ärztezeitung 2002;83[6]:257) hat das BSV am 11. Juli 2002 weitere Empfehlungen betreffend Vergünstigungen im Arzneimittelmarkt nachgeschoben. Deren Qualität hat sich nicht verbessert, im Gegenteil. Wir drucken die Empfehlungen trotzdem ab und beschränken uns an dieser Stelle auf folgende Feststellungen:

1. Das Ergebnis entspricht weder der Diskussion noch irgendeinem Beschluss der speziell zu diesem Zweck einberufenen Arbeitsgruppe «Rabatte und Boni», in der auch die FMH vertreten war. Das BSV hat die Empfehlungen nach einer abrupten Auflösung der Arbeitsgruppe in völliger Eigenregie formuliert.

2. Die Empfehlungen äussern sich sowohl zur Umsetzung des Weitergabegebotes von Art. 56 Abs. $3 \mathrm{KVG}$ als auch zum Vergünstigungsverbot von Art. 33 HMG. Dafür ist das BSV von vorneherein nicht zuständig: Die Strafverfolgung wegen Verstössen gegen Art. 33 HMG fällt in den Kompetenzbereich von Swissmedic.

Zudem handelt es sich um sogenannte Verwaltungsrichtlinien, an die der Richter grundsätzlich nicht gebunden ist. Sicher, solche Verwaltungsrichtlinien können insofern eine rechtliche Bedeutung erhalten, als Betroffene, die sich daran halten, im Endeffekt kaum Sanktionen zu befürchten haben. Die vorliegenden Empfehlungen des BSV sind indes derart schwammig, widersprüchlich und unpraktikabel, dass sie diese an sich wünschenswerte Leitlinienfunktion gar nicht erst entfalten können.

3. Unabhängig von den formalrechtlichen Einwänden kranken die Empfehlungen daran, dass sie den grundlegenden Widerspruch zwischen HMG (Vergünstigungsverbot) und KVG (Vergünstigungsweitergabe) nicht lösen und wohl auch nicht lösen können. Einfach ausgedrückt: Was verboten ist, kann auch nicht weitergegeben werden. Die einzig vernünftige Empfehlung wäre an sich diejenige an den Gesetzgeber, sich zu entscheiden, welches System er will:
- auf Verboten beruhend, mit dem Vorteil, dass pekuniäre Beeinflussungsversuche praktisch ausgeschlossen werden können, aber mit dem Nachteil, dass die staatlich administrierten Preise noch härter betoniert werden als zu Zeiten des Sanphar-Kartells;

- auf Wettbewerbselementen aufbauend, verknüpft mit (nicht einfach zu definierenden) Instrumenten zur Schaffung von Transparenz und entsprechender Preisgestaltung. Ausgedient hätte in einem solchen System freilich auch das sonntagsschülerhafte Weitergabegebot, wenigstens soweit es sich individuell an die einzelnen "Marktteilnehmer» richtet. Gefordert wären die Behörden, welche bei der Preisfestlegung mit dem Marktgeschehen mithalten müssten.

Fazit: So wie die Dinge nun stehen, werden sich alle Beteiligten im eigenen Interesse am Vergünstigungsverbot des HMG orientieren. Das Weitergabegebot des KVG wird toter Buchstabe bleiben.

Der Spielraum im HMG ist relativ eng, die Rechtsunsicherheit aber nach wie vor nicht behoben (Stichwort: «handelsübliche Rabatte»). Die lakonische Feststellung, es bleibe wohl oder übel der Rechtssprechung im Einzelfall vorbehalten, die Grenzen des Erlaubten zu ziehen, ist angesichts der strafrechtlichen Sanktionsdrohungen nicht gerade jedermanns Sache. Niemand wird Ihnen verübeln, wenn Sie dieses Risiko nicht selber tragen wollen; am besten wenden Sie sich für die Beurteilung eines konkreten Falles an Swissmedic oder an das BSV und lassen sich die Antwort schriftlich geben.

Darüber hinaus wird sich die Ärzteschaft, ebenso wie alle anderen Betroffenen, überlegen müssen, ob sie sich nun selbst eine einfache, alltagstaugliche Leitlinie für die Abläufe im Arzneimittelhandel geben will. Bleibt die Frage offen, ob Verbände, die in diese Richtung aktiv werden, dann nicht wieder von der Wettbewerbskommission als Kartelle abgestraft werden ... 